\title{
QCD and Natural Philosophy
}

\author{
Frank Wilczek \\ Center for Theoretical Physics \\ Massachusetts Institute of Technology \\ Cambridge, MA 02139-4307 \\ MIT-CTP \# 3328
}

Plenary talk at TH2002, Paris, July 2002.

\begin{abstract}
QCD sheds considerable light on several of the most basic features of the natural world, including the origin of mass, the feebleness of gravity, the extent to which the properties of matter can be determined conceptually, the possible utility of the anthropic principle, and the metatheoretic notions of effectiveness and computability. I discuss these applications here.
\end{abstract}


Contemporary academic or unnatural philosophy is characterized by extreme insistence on purity of abstract thought. A joke will illustrate the point.

A man walks into a bar, takes a seat on the next-to-last stool, and spends the evening chatting up the empty stool next to him, being charming and flirtatious, as if there were a beautiful woman in that empty seat. The next night, same story. And the next night, same story again. Finally the bartender can't take it any more. She asks, "Why do you keep talking to that empty stool as if there were a beautiful woman in it?"

The man answers, "I'm a philosopher. Hume taught us that it's logically possible that a beautiful woman will suddenly materialize on that stool, and no one has ever refuted him. If one does appear, then obviously I'll seem very clever indeed, and I'll have the inside track with her."

"That's ridiculous," says the bartender, who happens to be a physicist. "Plenty of very attractive women come to this bar all the time. You're reasonably presentable, and extremely articulate; if you applied your charm on one of them, you might succeed."

"I thought about trying that," he replies, "but I couldn't prove it would work."

In this vein, there are a lot of very attractive questions that working physicists normally abandon to the philosophers, or to quasi-scientists who speculate in reckless disregard of the facts, just because the questions are broad and qualitative. We shouldn't. I'd like in this talk to take a stab at a few such questions. These are questions for which a physical theory firmly established only relatively recently, quantum chromodynamics or QCD, affords us considerable new insight. I will not waste words introducing the technical content of QCD, nor defending its validity, as this background information is readily available in many places [1].

\section{What is the Origin of Mass?}

\subsection{Framing the Question}

That a question makes grammatical sense does not guarantee that it is answerable, or even coherent. In that spirit, let us begin with a critical examination of the question posed in this Section: What is the origin of mass?

In classical mechanics mass appears as a primary concept. It was a very great step for the founders of classical mechanics to isolate the scientific concept of mass. In Newton's laws of motion, mass appears as an irreducible, intrinsic property of matter, which relates its manifest response (acceleration) to an abstract cause (force). An object without mass would not know how to move. It would not know, from one moment to the next, where in space it was supposed to be. It would be, in a very strong sense, unphysical. Also in Newton's law of gravity, the mass of an object governs the strength of the force it exerts. One cannot build up an object that gravitates out of material that does not. Thus it is difficult to imagine, in the Newtonian framework, 
what could possibly constitute an "origin of mass". In that framework, mass just is what it is.

Later developments in physics make the concept of mass seem less irreducible. The undermining process started in earnest with the theories of relativity. The famous equation $E=m c^{2}$ of special relativity theory, written that way, betrays the prejudice that we should express energy in terms of mass. But we can also read it as $m=E / c^{2}$, which suggests the possibility of explaining mass in terms of energy. In general relativity the response of matter to gravity is independent of mass (equivalence principle), while space-time curvature is generated directly by energy-momentum, according to $R_{\mu \nu}-\frac{1}{2} g_{\mu \nu} R=\kappa T_{\mu \nu}$, with $\kappa \equiv 8 \pi G_{N} / c^{2}$. Mass appears as a contributing factor to energy-momentum, but it has no uniquely privileged status.

At an abstract level, mass appears as a label for irreducible representations of the Poincaré group. Since representations with $m \neq 0$ appear in tensor products of $m=0$ representations, it is possible, at least kinematically, to build massive particles as composites of massless particles, or of massless particles and fields.

\subsubsection{Lorentz's Dream}

At a much more concrete level, the question of the origin of mass virtually forced itself upon physicists' attention in connection with the development of electron theory. Electrons generate electromagnetic fields; these fields have energy and therefore inertia. Indeed, a classical point-electron is surrounded by an electric field varying as $e / r^{2}$. The energy in this field is infinite, due to a divergent contribution around $r \rightarrow 0$. It was a dream of Lorentz (pursued in evolved forms by many others, including Poincaré, Dirac, Wheeler, and Feynman), to account for the electron's mass entirely in terms of its electromagnetic fields by using a more refined picture of electrons. Lorentz hoped that in a correct model of electrons they would emerge as extended objects, and that the energy in the Coulomb field would come out finite, and account for all (or most) of the inertia of electrons.

Later progress in the quantum theory of electrons rendered this program moot by showing that the charge of an electron, and therefore of course its associated electric field, is intrinsically smeared out by quantum fluctuations in its position. Indeed, due to the uncertainty principle the picture of electrons as ideal point-particles certainly breaks down for distances $r \lesssim \hbar / m c$, the Compton radius. At momenta $p \gtrsim \hbar / r$, the velocity $p / m$ formally becomes of order $c$, and one cannot regard the electron as a static point source. If we cut off the simple electrostatic calculation at the Compton radius, we find an electromagnetic contribution to the electron mass of order $\delta m \sim \alpha m$, where $\alpha=e^{2} / 4 \pi \hbar c \approx 1 / 137$ is the fine structure constant. In this sense the easily identifiable and intuitive electromagnetic contribution to the mass, which Lorentz hoped to build upon, is small. To go further, we cannot avoid considering relativity and quantum mechanics together. That means quantum field theory. 


\subsubsection{Its Debacle}

In quantum electrodynamics itself, the whole issue of the electromagnetic contribution to the electron mass becomes quite dodgy, due to renormalization.

Quantum electrodynamics does not exist nonperturbatively. One can regulate and renormalize order-by-order in perturbation theory, but there are strong arguments that the series does not converge, or even represent the asymptotic expansion of a satisfactory theory. In a renormalization group analysis, this is because the effective coupling blows up logarithmically at short distances, and one cannot remove the cutoff. In a lattice regularization, one could not achieve a Lorentz-invariant limit. So one cannot strictly separate the issue of electromagnetic mass from the unknown physics which ultimately regularizes the short-distance singularities of QED.

If we regard QED as an effective theory, essentially by leaving in an energy cutoff $\Lambda$, corresponding to a distance cutoff $\hbar c / \Lambda$, we get a contribution to the mass at short distances going as $\delta m \propto \alpha m \log (\Lambda / m)$. Quantum mechanics has changed the powerlaw divergence into a logarithm. As a result, $\delta m$ is a fractionally small contribution to the total mass, at least for sub-Planckian $\Lambda$ (i.e., $\Lambda \lesssim 10^{19} \mathrm{GeV}$ ). We know that QED ceases to be a complete description of physics, or even a well-isolated theory, far below such energies.

In any case, since the mass renormalization is multiplicative, an electron field whose bare quanta have bare mass is zero produces physics quanta with physical mass zero. Indeed, the massless version of QED has enhanced symmetry - chiral symmetry which is not destroyed by (perturbative) renormalization.

In short, very little seems to survive from Lorentz's original dream. I've described this fiasco in some detail, since it provides an instructive background to contrast with our upcoming considerations.

\subsubsection{Upping the Ante}

Quantum field theory changes how we view the question of the origin of mass dramatically. As we have seen, it quashes hope for a simple classical mechanistic explanation.

At a more profound level, however, quantum field theory makes the question of the origin of mass seem both better posed and more crucial. Very few such theories are known to exist nonperturbatively in four space-time dimensions. Even if we relax our standards to include renormalizable quantum field theories, i.e. those which are reasonably self-contained as effective field theories, we find a very restricted set. Such theories, unless they contain many fields and few symmetries, allow few continuous

\footnotetext{
${ }^{1}$ Actually this blow-up, the famous Landau pole, arises from extrapolating the perturbative result beyond its range of validity. What one can deduce simply and rigorously is that the effective coupling does not become small at short distances: QED is not asymptotically free. If there is a fixed point at finite coupling, it may be possible to construct a relativistically invariant limiting theory. But even if such a theory were to exist, its physical relevance would be quite dubious, since we know that there's much more to physics than electrodynamics at distances so short that the logarithms matter.
} 
parameters. Among those few, parameters specifying bare masses of the quanta feature prominently. So massless theories are even more tightly constrained, and bring us closer to the ideal of a parameter-free, purely conceptual description of Nature.

Moreover, theories often acquire enhanced symmetry when mass parameters vanish. In such cases masslessness is "natural", in the sense that it can be stated as an abstract structural property of the theory, namely its enhanced symmetry. To the extent we feel a more symmetric theory is more beautiful, the massless theories are singled out as more beautiful. These features of naturalness and beauty, which motivate massless theories, survive even if one is not committed to the technical requirement of renormalizability.

\subsection{Most of the Answer: QCD Lite}

Enough of generalities! I want now to describe some very specific, and I think quite beautiful, insights into the origin of mass in the real world. We will construct following Nature - mass without mass, using only $c$ and $\hbar$.

\subsubsection{Introducing QCD Lite}

My central points are most easily made with reference to a slight idealization of QCD I call, for reasons that will be obvious, QCD Lite. It is a nonabelian gauge theory based on the gauge group $S U(3)$ coupled to two triplets and two antitriplets of lefthanded fermions, all with zero mass. Of course I have in mind that the gauge group represents color, and that one set of triplet and antitriplet will be identified with the quark fields $u_{L}, u_{R}$ and the other with $d_{L}, d_{R}$.

Upon demanding renormalizability, [2 this theory appears to contain precisely one parameter, the coupling $g$. It is, in units with $\hbar=c=1$, a pure number. I'm ignoring the $\theta$ parameter, which has no physical content here, since it can be absorbed into the definition of the quark fields. Mass terms for the gluons are forbidden by gauge invariance. Mass terms for the quarks are forbidden by chiral $S U(2)_{L} \times S U(2)_{R}$ flavor symmetry.

\subsubsection{Running Coupling; Dimensional Transmutation}

The coupling constant $g$ that appears in the Lagrangian of QCD Lite, like the corresponding constant $e$ in QED, is a dimensionless number (in units with $\hbar=c=1$ ). Likewise for the fine-structure constant $\alpha_{s} \equiv g^{2} / 4 \pi$. But the real situation, when we take into account the effect of quantum mechanics, is quite different. Empty space is a medium, full of virtual particles, and responds dynamically to charges placed within it. It can be polarized, and the polarization clouds surrounding test charges

\footnotetext{
${ }^{2} \mathrm{Or}$, in physical terms, the existence of a relativistically invariant limiting theory. Or alternatively, radical decoupling from an unspecified, gratuitous high-energy cutoff.
} 
can shield (screen) or enhance (antiscreen) their strength. In other words, quantummechanically the measured strength of the coupling depends on the distance scale, or equivalently the (inverse) energy scale, at which it is measured: $\alpha_{s} \rightarrow \alpha_{s}(Q)$. This is a central feature of QCD Lite, and of course of QCD itself. These theories predict that the effective coupling gets small at large $Q$, or equivalently at short distance.

This behavior displays itself in a remarkably direct and tangible form in the final states of electron-positron annihilation. Hadrons emerging from high-energy electronpositron annihilation organize themselves into collimated jets. Usually there are two jets, but occasionally three. The theoretical interpretation is profound but, given asymptotic freedom, straightforward. The primary products emerging from the annihilation are a quark and an antiquark. They emit soft - that is, low energy-momentum - radiation copiously, but only rarely hard radiation. That's a restatement, in momentum space, of asymptotic freedom. The soft radiation materializes as many particles, but these particles inherit their direction of flow from the quark or antiquark, and therefore constitute a jet. In the relatively rare case that there is hard radiation, that is to say, emission of an energetic gluon, the gluon induces its own independent jet. All this can be made completely quantitative. There are precise predictions for the ratio of three- to two-jet events, the rare occurrence of four (or more) jet events, how these ratios change with energy, angular dependence, and so forth. The observations agree with these predictions. Thus they provide overwhelming, direct evidence for the most basic elements of the theory, that is the quark-gluon and gluon-gluon couplings.

Because the coupling runs, we can, within any given version of QCD Lite, measure any given numerical value $a=\alpha_{s}(Q)$, simply by choosing an appropriate $Q$. It appeared, classically, that we had an infinite number of different versions of QCD Lite, with different values of the coupling parameter. In reality the only difference among all these theories, after they are quantized, is the unit they choose for measuring mass. All dimensionless physical parameters, and in particular all mass ratios, are uniquely determined. We can trade the dimensionless parameter $g$ for the unit of mass. This is the phenomenon of dimensional transmutation.

Of course the value of the overall energy scale makes a big difference when we come to couple QCD Lite, or of course QCD, to the rest of physics. Gravity, for example, cares very much about the absolute value of masses. But within QCD Lite itself, if we compute any dimensionless quantity whatsoever we will obtain a unique answer, independent of any choice of coupling parameter. Thus, properly understood, the value of the QCD coupling constant does not so much govern QCD itself - within its own domain, QCD is unique - but rather how QCD fits in with the rest of physics.

\subsubsection{Physical Mass Spectrum - QCD Lite and Reality}

Now let us consider more concretely how these dynamical phenomena lead us to a nontrivial hadron spectrum. Looking at the classical equations of QCD, one would expect an attractive force between quarks that varies with the distance as $g^{2} / 4 \pi r^{2}$, where $g$ is the coupling constant. This result is modified, however, by the effects 
of quantum fluctuations. As we have just discussed, the omnipresent evanescence of virtual particles renders empty space into a dynamical medium, whose response alters the force law. The antiscreening effect of virtual color gluons (asymptotic freedom), enhances the strength of the attraction, by a factor which grows with the distance. This effect can be captured by defining an effective coupling, $g(r)$, that grows with distance.

The attractive interaction among quarks wants to bind them together; but the potential energy to be gained by bringing quarks together must be weighed against its cost in kinetic energy. In a more familiar application, just this sort of competition between Coulomb attraction and localization energy is responsible for the stability and finite size of atoms. Here quantum-mechanical uncertainty implies that quark wave-functions localized in space must contain a substantial admixture of high momentum components. For a relativistic particle, this translates directly into energy. If the attraction followed Coulomb's law, with a small coupling, the energetic price for staying localized would always outweigh the profit from attraction, and the quarks would not form a bound state. Indeed, the kinetic energy $\hbar c / r$ beats the potential energy $g^{2} / 4 \pi r$. But the running coupling of QCD grows with distance, and that tips the balance. The quarks finally get reined in, at distances where $\alpha_{s}(r)$ becomes large.

We need not rely on heuristic pictures, or wishful thinking, to speculate about the mass spectrum of QCD Lite. It has been calculated by direct numerical integration of the fundamental equations, using the techniques of lattice gauge theory. The results bear a remarkable qualitative and semiquantitative resemblance to the observed spectrum of nonstrange hadrons, generally at the $10 \%$ level, comfortably within known sources of error due to finite size, statistics, etc. - and (anticipating) small quark masses. Of course, in line with our preceding discussion, the overall scale of hadron masses is not determined by the theory. But all mass ratios are predicted, with no free parameters, as of course are the resonance quantum numbers.

QCD Lite is not the real world, of course. So although in QCD Lite we get mass without mass in the strict sense, to assess how much real-world mass arises this way we need to assess how good an approximation QCD Lite is to reality, quantitatively. We can do this by adjusting the nonzero values of $m_{u}$ and $m_{d}$ to make the spectrum best fit reality, and then seeing how much they contributed to the fit.f Unlike charges in QED, masses in QCD are soft perturbations, and we can calculate a meaningful finite difference between the spectra of these two theories. There is also a well-developed alternative approach to estimating the contribution of quark masses by exploiting the phenomenology of chiral symmetry breaking. Either way, one finds that the quark masses contribute at most a few per cent to the masses of protons and neutrons.

\footnotetext{
${ }^{3}$ There are significant technical issues around realizing chiral symmetry in numerical work involving discretization on a lattice. Recent theoretical work appears to have resolved the conceptual issues, but the numerical work does not yet fully reflect this progress. To avoid a fussy presentation I've oversimplified by passing over these issues, which do not affect my main point.

${ }^{4}$ Again, there are significant technical issues here, especially regarding the role of the strange quark. Fortunately, the uncertainties are numerically small.
} 
Protons and neutrons, in turn, contribute more than $99 \%$ of the mass of ordinary matter. So QCD Lite provides, for our purpose, an excellent description of reality. The origin of the bulk of the mass of ordinary matter is well accounted for, in a theory based on pure concepts and using no mass parameters - indeed, no mass unit - at all!

\subsubsection{Comparing with the Old Dream}

While our final result fulfills the fantasy of Lorentz's dream, the details and the mechanism whereby it is achieved are quite different from what he imagined.

Obviously we are speaking of hadrons, not electrons, and of QCD, not classical electrodynamics. The deepest difference, however, concerns the source and location of the energy whereby $m=E / c^{2}$ is realized. In Lorentz's dream, the energy was self-energy, close to the location of the point particle. In QCD Lite the self-mass vanishes. Paradoxically, there is a sense in which the self-energy of a quarkf is infinite (confinement), but this is due to the spatial extent of its color field, which has a tail extending to infinity, not to any short-distance singularity. To make physical hadrons, quarks and gluons must be brought together in such a way that the total color vanishes. Then there is no infinite tail of color flux. The different tails of the individual quarks and gluons have annulled one another. But at finite distances the cancellation is incomplete, because Heisenberg's uncertainty principle imposes an energetic cost for keeping color charges precisely localized together. The bulk of the mass of the hadrons comes from the residues of these long tails, not from singularities near point-like color charges.

\subsubsection{Comparing with the Old Debacle}

It is instructive to review, point by point, how the obstructions that quantum field theory appeared to pose for the "Origin of Mass" or "Mass without Mass" program are resolved in QCD.

First of all QCD does exist even nonperturbatively, so the issues connected with renormalization can be analyzed consistently down to rock bottom.

Second, the massless theory is "natural" in the technical sense defined above. It displays enhanced symmetry. The gluons have no mass parameter because there is no such parameter consistent with local gauge symmetry. For quarks, there is an additional chiral $S U(2)_{L} \times S U(2)_{R}$ symmetry, expanded from the vectorial $S U(2)_{L+R}$, when they massless.

Third, these symmetries, which guarantee zero bare mass for the bare quanta, do not forbid nonzero mass for physical quanta. The gauge symmetry acts trivially on the physical quanta (confinement). The chiral symmetry is spontaneously broken: it

\footnotetext{
${ }^{5}$ Infinite self-energy does not conflict with zero mass. $E=m c^{2}$ describes the energy of a particle of mass $m$ when it is at rest; but of course, as we know from photons, there can also be energy in massless particles, which cannot be brought to rest.
} 
is a property of the underlying equations but not of their stable solution (i.e., of their physical realization). Spontaneously broken chiral symmetry predicts the existence of massless collective modes, the Nambu-Goldstone bosons - pions - along with many of their properties; but it does not forbid mass for the other hadrons - protons, neutrons, etc.

Fourth, the realistic theory, with small nonzero quark masses, is a soft perturbation of the massless theory. It does not require that we make any alteration in the renormalization procedure. Thus it is commensurable with the massless theory. We can compare them unambiguously and quantitatively.

Fifth, and finally, this quantitative comparison shows that the nonzero quark masses in Nature contribute only a small fraction to the masses of protons and neutrons. The chiral symmetry of the massless theory is approximately valid in reality, and is the basis of a very useful phenomenology. The mass ratios of hadrons computed in the idealized massless theory are very nearly the same as in reality (apart, of course, from the pions).

\section{3 (Many) Remaining Issues}

While the dynamical energy of massless QCD accounts for the bulk of mass in ordinary matter, it is far from providing the only source of mass in Nature.

Mass terms for quarks and charged leptons appear to violate the electroweak gauge symmetry $S U(2) \times U(1)$. But gauge symmetry cannot be violated in the fundamental equations - that would lead to ghosts and/or nonunitarity, and prevent construction of a sensible quantum theory. So these masses must, in a sense, have their "origin" in spontaneous symmetry breaking. That is accomplished, in the Standard Model, by having a nonsinglet Higgs field acquire a vacuum expectation value. Why this value is so small, compared to the Planck scale, is one aspect of what is usually called the hierarchy problem. Why the couplings of this field are so disparate - especially, what is particularly crucial to the structure of physical reality, why its dimensionless couplings to $e, u, d$ are so tiny (in the range $10^{-5}$ to $10^{-6}$ ) - is an aspect of what is usually called the flavor problem.

Then there are separate problems for generating masses of supersymmetric particles (soft breaking parameters, $\mu$ term), for generating the mass of cosmological 'dark matter' (this might be included in the previous item!), for generating neutrino masses, and now apparently for generating the mass density of empty space (cosmological term).

Obviously, many big questions about the origin of mass remain. But I think we've answered a major one, the one that is most important quantitatively for ordinary matter, beautifully and convincingly. 


\section{Why is Gravity Feeble?}

Gravity dominates the large-scale structure of the Universe, but only by default. Matter arranges itself to cancel electromagnetism, and the strong and weak forces are intrinsically short-ranged. At a more fundamental level, gravity is extravagantly feeble. Acting between protons, gravitational attraction is about $10^{-36}$ times weaker than electrical repulsion. Where does this outlandish disparity from? What does it mean?

Feynman wrote

There's a certain irrationality to any work on [quantum] gravitation, so it's hard to explain why you do any of it .... It is therefore clear that the problem we working on is not the correct problem; the correct problem is What determines the size of gravitation?

I want to argue that in modern physics it is natural to view the problem of why gravity is extravagantly feeble in a new way - upside-down and through a distorting lens compared to its superficial appearance. When viewed this way, it comes to seem much less enigmatic.

First let me quantify the problem. The mass of ordinary matter is dominated by protons (and neutrons), and the force of gravity is proportional to (mass) ${ }^{2}$. From Newton's constant, the proton mass, and fundamental constants we can form the pure dimensionless number

$$
X=G_{N} m_{p}^{2} / \hbar c
$$

where $G_{N}$ is Newton's constant, $m_{p}$ is the proton mass, $\hbar$ is Planck's constant, and $c$ is the speed of light. Substituting the measured values, we obtain

$$
X \approx 6 \times 10^{-39} .
$$

This is what we mean, quantitatively, when we say that gravity is extravagantly feeble.

We can interpret $X$ directly in physical terms, too. Since the proton's geometrical size $R$ is roughly the same as its Compton radius $\hbar / m_{p} c$, the gravitational binding energy of a proton is roughly $G_{N} m_{p}^{2} / R \approx X m_{p} c^{2}$. So $X$ is the fractional contribution of gravitational binding energy to the proton's rest mass!

\subsubsection{Planck's Hypothesis}

An ultimate goal of physical theory is to explain the world purely conceptually, with no parameters at all. Superficially, this idea seems to run afoul of dimensional analysis - concepts don't have units, but physical quantities do!

There is a sensible version of this goal, however, that is rapidly becoming conventional wisdom, despite falling well short of scientific knowledge. Soon after he introduced his constant $\hbar$, in the course of a phenomenological fit to the black-body radiation spectrum, Planck pointed out the possibility of building a system of units 
based on the three fundamental constants $\hbar, c, G_{N}$. Indeed, from these three we can construct a unit of mass $\left(\hbar c / G_{N}\right)^{1 / 2}$, a unit of of length $\left(\hbar G_{N} / c^{3}\right)^{1 / 2}$, and a unit of time $\left(\hbar G_{N} / c^{5}\right)^{1 / 2}$ - what we now call the Planck mass, length, and time respectively.

Planck's proposal for a system of units based on fundamental physical constants was, when it was made, rather thinly rooted in physics. But by now there are profound reasons to regard $c, \hbar$ and $G$ as conversion factors rather than numerical parameters. In the special theory of relativity there are symmetries relating space and time - and $c$ serves as a conversion factor between the units in which space-intervals and timeintervals are measured. In quantum theory, the energy of a state is proportional to the frequency of its oscillations - and $\hbar$ is the conversion factor. Thus $c$ and $\hbar$ appear directly as measures in the basic laws of these two great theories. Finally, in general relativity theory space-time curvature is proportional to the density of energy - and $G_{N}$ (actually $G_{N} / c^{4}$ ) is the conversion factor.

If we want to adopt Planck's astonishing hypothesis that we must build up physics solely from these three conversion factors, then the enigma of $X$ 's smallness looks quite different. We see that the question it poses is not "Why is gravity so feeble?" but rather "Why is the proton's mass so small?" For according to Planck's hypothesis, in natural (Planck) units the strength of gravity simply is what it is, a primary quantity. So it can only be the proton's mass that provides the tiny number $\sqrt{X}$.

\subsubsection{Running in Place}

That's a provocative and fruitful way to invert the question, because we now have a quite deep understanding of the origin of the proton's mass, as I've reviewed above.

The proton mass is determined, according to the dynamics I've described, by the distance at which the running QCD coupling becomes strong. Let's call this the QCDdistance. Our question, "Why is the proton mass so small?" has been transformed into the question, "Why is the QCD-distance much larger than the Planck length?" To close our circle of ideas, we need to explain how it is that, if only the Planck length is truly fundamental, such a vastly different length arises naturally.

This last elucidation, profound and beautiful, is worthy of the problem. It has to do with how the coupling runs, in detail. When the QCD coupling is weak, "running" is actually a bit of a misnomer. Rather, the coupling creeps along like a wounded snail. We can in fact calculate the behavior precisely, following the rules of quantum field theory, and even test it experimentally, as I mentioned before. The inverse coupling varies logarithmically with distance. So if we want to evolve an even moderately small coupling into a coupling of order unity, we must let it run between length-scales whose ratio is exponentially large. So if the QCD coupling is even moderately small at the Planck length it will reach unity only at a much larger distance.

Numerically, what we predict is that $\alpha_{s}\left(l_{\mathrm{Pl}}\right)$ at the Planck length is roughly a third to a fourth of what it is observed to be at $10^{-15} \mathrm{~cm}$; that is, $\alpha_{s}\left(l_{\mathrm{Pl}}\right) \approx 1 / 30$. We cannot measure $\alpha_{s}\left(l_{\mathrm{Pl}}\right)$ directly, of course, but there are good independent reasons, having to do with the unification of couplings, to believe that this value holds in 
reality. It is amusing to note that in terms of the coupling itself, what we require is $g_{s}\left(l_{\mathrm{Pl}}\right) \approx 1 / 2$ ! From this modest and seemingly innocuous numerical hypothesis, which involves neither really big numbers nor speculative dynamics beyond what is supported by hard experimental evidence, we have produced a logical explanation of the tiny value of $X$.

\section{The Reduction of Matter}

The reductionist program, roughly speaking, is to build up the description of Nature from a few laws that govern the behavior of elementary entities, and that can't be derived from anything simpler. This definition is loose at both ends.

On the output side, because in the course of our investigations we find that there are important facts about Nature that we have to give up on predicting. These are what we - after the fact! - come to call contingencies. Three historically important examples of different sorts are the number of planets in the Solar System, the precise moment that a radioactive nucleus will decay, or what the weather will be in Boston a year from today. In each of these cases, the scientific community at first believed that prediction would be possible. And in each case, it was a major advance to realize that there are good fundamental reasons why it is not possible.

On the input side, because it is difficult - perhaps impossible - ever to prove the non-existence of simpler principles. I'll revisit this aspect at the end of the lecture.

Nevertheless, and despite its horrible name, the reductionist program has been and continues to be both inspiring and astoundingly successful. Instead of trying to refine an arbitrary a priori definition of this program, it is more edifying to discuss its best fruits. For beyond question they do succeed to an astonishing extent in "reducing" matter to a few powerful abstract principles and a small number of parameters.

It is most instructive to build up to our most complete reduction of matter from some intermediate models that are extremely important and useful in their own right.

\subsection{Structural Chemistry}

The first such model is obtained by regarding both nuclear masses and the speed of light as effectively infinite. We then have the theory of nonrelativistic electrons in the presence of highly localized, static sources of positive charge, in multiples of $|e|$, where $e$ is the electron charge. We compute the energy as a function of the positions of the source charges, using the Schrödinger equation. Local minima define molecules, and we can compute their wavefunctions. This model gives a good approximation for the vast subject of structural chemistry. It Its only continuous parameters are $\hbar, e$, and the electron mass $m_{e}$. Since $\hbar$ can be considered as a conversion factor, and

\footnotetext{
${ }^{6}$ There is an additional fine point, that we must specify some symmetry rules to take account of quantum statistics for identical nuclei.
} 
the Bohr radius $\hbar^{2} / m_{e} e^{2}$ just sets the overall length scale, this is essentially a oneparameter theory. Indeed, since one cannot manufacture a dimensionless quantity out of $\hbar, e, m_{e}$, the remaining parameter is really a conversion factor too. Thus we have a zero-parameter theory of structural chemistry!

A more accurate version - QED with sources - recognizes $c \neq \infty$ and brings in real and virtual photons. Now there is a pure number in the game, namely of course the fine structure constant $e^{2} / 4 \pi \hbar c$. Regarding $\hbar$ and $c$ as conversion factors, and taking into account that $\hbar / m_{e} c$ can be used to define the overall length scale, we arrive at a very accurate one-parameter theory of structural chemistry and photochemistry.

\subsection{Matter}

The shortcoming of this theory, of course, is that the matter doesn't move (except by radiative transitions). The theory is missing vibrational and rotational levels, not to mention reactions, diffusion, etc.

To allow motion, we must put in finite values for the nuclear masses. In doing this, we open ourselves up to many, many more input parameters. At a minimum we need the masses of the different nuclei and isotopes, and for accuracy in details we need their spins and magnetic moments, etc. At this level, all of them must be taken from experiment. There are several hundreds of parameters. Nevertheless, because they are highly overconstrained by hundreds of thousands of measurements, the resulting theory is extremely useful. It is the foundation of practical quantum chemistry.

The next step in reduction is the one supplied by QCD. QCD assures us that all the nuclear parameters can be calculated crudely - perhaps at the $10 \%$ level using QCD Lite. To do this, we need only one parameter beyond $\hbar$ and $c$, as I've already discussed. That parameter can be taken as the coupling $g_{s}$, normalized at some appropriate energy scale (say $10^{5} m_{e} c^{2}$ ), or equivalently the energy scale $\Lambda_{\mathrm{QCD}}$, which is roughly speaking the scale at which the coupling becomes large. To do a more accurate job, we also need to introduce the light quark masses $m_{u}, m_{d}$.

Our faith in QCD is not based primarily on its practical ability to reproduce nuclear parameters. The best quantitative tests of the theory come from an entirely different domain of behavior, scattering at ultra-high energy, where the quark and gluon degrees of freedom and their couplings are clearly revealed. We rely on the successful outcome of these tests, and (so far) rather crude results from massive numerical calculations of proton structure and the hadron spectrum for empirical support. This support is highly leveraged by the theory's being rigidly principled. It embodies the basic principles of relativity, quantum mechanics, and gauge symmetry. Only a few parameters appear in the most general consistent embodiment of these principles. They are just the ones we use!!

So in practice the "reduction" we offer the chemists, or for that matter the nuclear physicists, is not of much use to them. But it is there, as a matter of principle.

\footnotetext{
${ }^{7}$ There is also the notorious $\theta$ parameter, a can of worms I don't want to dive into just now.
} 
At this point our best available reduction of ordinary matter is in place. A fundamental theory that we believe offers an extremely complete and accurate set of equations governing the structure and behavior of matter in ordinary circumstances, with a very liberal definition of "ordinary", requires the parameters $\hbar, c, e, m_{e}, \Lambda_{\mathrm{QCD}}, m_{u}, m_{d}$. Among these $\hbar$ and $c$ can be regarded as conversion factors, $m_{u}$ and $m_{d}$ are relatively minor players, and one combination just sets an overall length scale. Thus we have a rough reduction of matter involving two parameters, and an accurate one involving four. Not bad!

\subsection{Astrophysics}

In astrophysics, as opposed to condensed matter physics, chemistry, and biology, gravity and the weak interactions play important roles. Their intrinsic weakness is compensated by the availability of vast amounts of matter and vast amounts of time, respectively. To treat these additional interactions we must introduce two additional parameters, the Newton constant $G_{N}$ and the Fermi constant $G_{F}$. Altogether, then, we now have $\hbar, c, e, m_{e}, \Lambda_{\mathrm{QCD}}, m_{u}, m_{d}, G_{N}, G_{F}$. Referring back to our discussion of Planck's hypothesis, we are inclined to regard $\hbar, c$ and $G_{N}$ as conversion factors. Also, we derive both $e$ and $\Lambda_{\mathrm{QCD}}$ from the value of the unified coupling at the Planck scale. So we are left with this "reasonable" number $\left(g_{\text {unified }} \approx 1 / 2\right)$ and four "unreasonable" numbers: $m_{e}, m_{u}, m_{d}$, and $G_{F}^{-\frac{1}{2}}$, that specify various masses in Planck units.

The absurd smallness of these numbers, and of the dimensionless ratio $m_{e}\left(G_{F} / \hbar c\right)^{\frac{1}{2}}$, define important unmet challenges for theoretical physics. They are salient aspects of what are called the hierarchy and flavor problems, respectively.

Though wonderful challenges remain, the success of the reductionist program is already most impressive. An accurate description of matter, including astrophysics, is built up from a few abstract principles and five parameters.

\subsection{Cosmology and Quantitative Anthropics}

Having come this far it would be inhuman not to take the discussion to its logical climax in cosmology. That brings in many unsettled questions that go far beyond the scope of this lecture. I'll restrict myself to a very brief discussion of a few issues closely tied to the foregoing considerations. I'll assume as given the emerging "standard model of cosmology", based on small perturbations around a spatially flat FriedmannRobertson-Walker model.

To specify quantitatively the standard model of cosmology we must add a few more parameters to our world-model. These include at least the baryon number density $\rho_{b}$, the dark matter density $\rho_{d}$, the overall amplitude $A$ of a scale-invariant spectrum of primordial fluctuations (assumed adiabatic and Gaussian), and the cosmological

\footnotetext{
${ }^{8}$ It is important to note that the dimensionless form of this ratio does not involve $G_{N}$. Its absurd smallness is not an artifact of Planck units.
} 
term $\Lambda$. Further work may uncover the need for additional parameters, for example if the Universe is not quite flat, or if the spectrum of perturbations is not quite scaleinvariant, but for now the four I mentioned appear to form an adequate, minimal set. If we accept that cosmology is "reduced" to general principles and four continuous parameters, beyond the ones we use to describe matter and astrophysics, the question arises how far this achievement fulfills the reductionist program. I think that most physicists do not feel entirely satisfied with it. That is because the parameters in cosmology, given in this way, do not appear directly in the description of the behavior of simple fundamental entities. Rather they appear as the description of average properties of macroscopic (very macroscopic!) agglomerations. We would like to calculate these parameters in terms of different ones more related to the fundamental laws of physics in the early Universe. There are rough ideas about how we might do this in the cases of $\rho_{b}$ (B and CP violation) and $\rho_{d}$ (axion and/or lightest supersymmetric particle production), even rougher ideas in the case of $A$ (inflationary potentials), and various tenuous speculations regarding $\Lambda$. There is a very good chance, I think, that the constitution of the dark matter will detected experimentally in the near future. Then we'll have a much better chance to understand at least $\rho_{d}$ properly!

Taken this far the status of the reductionist program in cosmology, though much less fully developed, is not very different in character from the preceding discussions of matter and astrophysics. But there are specifically cosmological issues, too.

Although it is usually passed over in silence, I think it is very important philosophically, and deserves to be emphasized, that our standard cosmology is radically modest in its predictive ambitions. It consigns almost everything about the world as we find it to contingency. That includes not only the aforementioned question of the number of planets in the Solar System, but more generally every specific fact about every specific object or group of objects in the Universe, apart from a few large-scale statistical regularities. Indeed, specific structures are supposed to evolve from the primordial perturbations, and these are only characterized statistically. In inflationary models these perturbations arise as quantum fluctuations, and their essentially statistical character is a consequence of the laws of quantum mechanics.

This unavoidably suggests the question whether we might find ourselves forced to become even more radically modest. If Let us suppose for the sake of argument the best possible case, that we had in hand the fundamental equations of physics. Some of my colleagues think they do, or soon will. Even then we have to face the question of what principle determines the solution of these equations that describes the observed Universe. Let me again suppose for the sake of argument the best possible case, that there is some principle that singles out a unique acceptable solution. Even then there is a question we have to face: If the solution is inhomogeneous, what determines our location within it?

Essentially inhomogeneous solutions of fundamental equations are by no means a contrived or remote possibility. They are at the heart of all the brane-world construc-

\footnotetext{
${ }^{9} \mathrm{~A}$ few outliers hope to move back the other way. One can only wish them luck!
} 
tions that have received much attention recently; they are characteristic of eternal inflation models; they arise very naturally in axion physics and its generalizations [1]. On a less cosmic scale, closer to home, they are what we see all around us - and of course, each of us is a highly structured inhomogeneity.

The standard cosmological model is yet another case, and supplies an especially clear parable. As we have just discussed, the laws of reductionist physics do not suffice to tell us about the specific properties of the Sun, or of Earth. Indeed, there are many roughly similar but significantly different stars and planets elsewhere in the same Universe. On the other hand, we can aspire to a rational, and even to some extent quantitative, "derivation" of the parameters of the Sun and Earth based on fundamental laws, if we define them not by pointing to them as specific objects - that obviates any derivation - but rather by characterizing broad aspects of their behavior. In principle any behavior will do, but possibly the most important and certainly the most discussed is their role in supporting the existence of intelligent observers, the so-called anthropic principle. There are many peculiarities of the Sun and Earth that can be explained this way. A crude example is that the mass of the Sun could not be much bigger or much smaller than it actually is because it would burn out too fast or not radiate sufficient energy, respectively.

Now if the Universe as we now know it constitutes, like the Solar System, an inhomogeneity within some larger structure, what might be a sign of it? 四 If the parameters of fundamental physics crucial to life - just the ones we've been discussing! - vary from place to place, and most places are uninhabitable, there would be a signature to expect. We should expect to find that some of these parameters appear very peculiar - highly nongeneric - from the point of view of fundamental theory, and that relatively small changes in their values would preclude the existence of intelligent observers. Weinberg has made a case that the value of the cosmological term $\Lambda$ fits this description; and I'm inclined to think that $\left(m_{u}-m_{d}\right) / \Lambda_{\mathrm{QCD}}$ and several other combinations of the small number of ingredients in our reduced description of matter and astrophysics do too. A fascinating set of questions is suggested here, that deserves careful attention [2].

\section{Metatheory}

QCD is unique among physical theories in its combination of logical closure and empirical success. So it is appropriate to consider it from the outside, as an object of metatheory.

\footnotetext{
${ }^{10}$ Of course, to make it interesting I'm assuming that we can't actually look beyond, due to the vastness of the homogeneous region we're in, or the limitations of our probes.
} 


\subsection{The Unreasonable Effectiveness of QCD}

There is a sense in which QCD is better than it has to be. It is not a complete theory of the world. Even within the standard model quarks have additional interactions, beyond QCD, that become significant - indeed, problematic - at short distances. So there is no logical requirement that QCD, extrapolated on its own down to infinitely short distances, should exist as a fully consistent relativistic quantum field theory. But it does. Is this just a happy coincidence, or does it point to something deeper? (Candidate idea: There must be fully consistent quantum field theories, because such theories are the ultimate description of Nature. A few years ago this might have been string-blasphemy; now it might be acceptable as duality.)

Although it's somewhat off the point, I'll take the opportunity to mention that this over-effectiveness reminds me of another apparently quite unrelated case, the cosmic censorship hypothesis in general relativity. We know that classical general relativity is not a complete theory, and there's no logical reason why it should hide its defects from view. Yet in many cases, and perhaps generically, it does seem that singularities get hidden behind horizons.

\subsection{The Unreasonable Ineffectiveness of QCD}

There some aspects of QCD I find deeply troubling - though I'm not sure if I should!

I find it disturbing that it takes vast computer resources, and careful limiting procedures, to simulate the mass and properties of a proton with decent accuracy. And for real-time dynamics, like scattering, the situation appears pretty hopeless. Nature, of course, gets such results fast and effortlessly. But how, if not through some kind of computation, or a process we can mimic by computation?

Does this suggest that there are much more powerful forms of computation that we might aspire to tap into? Does it connect to the emerging theory of quantum computers? These musings suggest some concrete challenges: Could a quantum computer calculate QCD processes efficiently? Could it defeat the sign problem, that plagues all existing algorithms with dynamical fermions? Could it do real-time dynamics, which is beyond the reach of existing, essentially Euclidean, methods?

Or, failing all that, does it suggest some limitation to the universality of computation?

Deeply related to this is another thing I find disturbing. If you go to a serious mathematics book and study the rigorous construction of the real number system, you will find it is quite hard work and cumbersome. QCD, and for that matter the great bulk of physics starting with classical Newtonian mechanics, has been built on this foundation. In practice, it functions quite smoothly. It would be satisfying, though, to have a "more reduced" description, based on more primitive, essentially discrete structures. Fredkin and recently Wolfram have speculated at length along these lines. I don't think they've got very far, and the difficulties facing such a program are immense. But it's an interesting issue. 


\section{Acknowledgments}

This work is supported in part by funds provided by the U.S. Department of Energy (D.O.E.) under cooperative research agreement \#DF-FC02-94ER40818. I'd like to thank the organizers for putting together a varied and fascinating program, and bringing it all to Paris. And I'd like specifically to thank Dominique Schiff for encouraging me to give a "philosophical" talk, which I had fun doing. She shouldn't be held responsible for my excesses, though.

\section{References}

[1] The material in Sections 1 and 2 largely overlaps corresponding parts of my contribution Four Big Questions With Pretty Good Answers to the Heisenberg centennial symposium, hep-ph/0201222, with some changes and additions. Also, the generalized axion cosmology alluded to in Section 3 is discussed there in much more detail.

[2] For some relevant discussion, and an extensive set of references, see C. Hogan, Reviews of Modern Physics 72 1149-1161 (2000). 